\title{
A Multi-Objectives Optimization to Develop the Mobile Dimension in a Small Private Online Course (SPOC)
}

\author{
Naima BELARBI ${ }^{1}$ \\ Abdelwahed NAMIR ${ }^{2}$ \\ Laboratory of Technological \\ Information and Modelisation \\ (LTIM) \\ Faculty of Sciences Ben M'Sik \\ University Hassan II \\ Casablanca \\ Morocco
}

\author{
Mohamed TALBI ${ }^{3}$ \\ Observatory of Research in Didactics \\ and University Pedagogy (ORDIPU) \\ Laboratory of Analytical Chemistry \\ and Physical Chemistry of Materials \\ Faculty of Sciences Ben M'Sik \\ University Hassan II \\ Casablanca \\ Morocco
}

\author{
Nadia Chafiq ${ }^{4}$ \\ Laboratory of Sciences, Information \\ Communication and Education \\ Technology (LAPSTICE) \\ Faculty of Sciences Ben M'Sik \\ University Hassan II \\ Casablanca \\ Morocco
}

\begin{abstract}
The impact of the mobile technology trend is being felt in several sectors today, including education. In this paper, we present an analysis of the development of the mobile dimension in a Massive Open Online Course (MOOC) or a Small Private Online Course (SPOC) as a decision-making problem among various approaches which cannot be ordered incontestably from the best to the worst. This is due to the fact that the various approaches to integrate the mobile dimension are different and that each solution presents both advantages and shortcomings from a technological point of view. The decision must be made on the basis of the end-users' requirements and usage. We propose to view this situation as a multi-objective optimization problem as the decision is a compromise between several conflicting objectives/criteria. The various approaches to the development of mobile access to a MOOC/SPOC are presented first and then compared using various criteria. Then we provide an analysis of the alternatives to find the non-dominant Pareto solutions.
\end{abstract}

Keywords-Mobile dimension; MOOC/SPOC; multi-objective optimization; criteria; decision

\section{INTRODUCTION}

Nowadays, the impact of the mobile technology trend is felt in several sectors including education. In fact, the majority of users use their smartphones or laptops more for various uses. MOOCs and SPOCs are also affected by this challenge in order to meet users' expectations in terms of mobile access. MOOCs and SPOCs providers must also align their platforms with the requirements of mobile devices and mobile users. Indeed, having an online learning tool accessible to everyone, usable anytime and anywhere, and being able to easily access information and resources online is a very important aspect of mobile online learning which opens a new way to consider, explore and develop in MOOCs/SPOCs.
In the mobile world, the most popular approaches to mobile development are:

- Responsive Web Designing (RWD)

- Designing a mobile website (web app)

- Designing a mobile application

- The design of a native mobile application

- The design of a hybrid mobile application

These approaches are different in nature and the choice between them depends on the field of application and other considerations.

We propose to view the situation as a multi-objective optimization problem as the decision is the compromise between several conflicting objectives/criteria.

A Multi-objective optimization analysis is mainly at the origin of the field of Economic Balance and theories of wellbeing [1], but its application to several fields is constantly expanding thanks in particular to advances in data analysis techniques and algorithms. In fact, this optimization analysis was used in multitask learning [2], for solving engineering problems [3], in the metal forming industry [4] and to optimize laminated composite structures [5].

In this paper, we have first explored work concerning the fusion of both environments: MOOCs, SPOCS and mobile learning. We then listed the various mobile strategies of the main MOOC providers. We have also presented the various possibilities for the integration of the mobile dimension in the MOOC/SPOC environments, pointing out the advantages and the disadvantages of every approach. A comparison analysis between these different approaches based on the main criteria involved in decision making for a mobile solution has been presented. 
Our main contribution is to demonstrate that the integration of the mobile dimension in a MOOC/SPOC is a multiobjective optimization problem. Indeed, it is not easy to find an "optimal" solution as the decision is a compromise among several conflicting objectives and this compromise is not perceived in the same way by all the decision-makers. This can therefore be considered as a multi-objective optimization problem. In the present work, we showed that the proposed four mobile solutions are an optimal set of non-dominant solutions since no improvements can be achieved for one objective without deteriorating the value of another one.

\section{RELATED WORKS}

\section{A. MOOCs/SPOCs and Mobile Dimension}

With the development of mobile technologies, MOOCs and SPOCs must meet this challenge in order to fulfill users' expectations in terms of mobile access. MOOC and SPOC providers also need to align their platforms to address the requirements of the mobile devices and users. The research that explored the combination of both learning formats, MOOCs and mobile learning, yielded some benefits, such as optimising the interaction and dialogue among learners, fostering collaborative learning, informal learning as well as lifelong learning [6] and the potential to extend the reach of MOOCs, improving access to learning materials and enabling new learning forms [7].

Choosing a strategy to integrate the mobile dimension into a MOOC/SPOC is not simple. Some research work provides some elements of comparison and feedback that can provide an idea of the different approaches to integrating the mobile dimension in a MOOC/SPOC.

In [8], the author reports that native or hybrid applications are no longer required to access a MOOC/SPOC that adheres to the principle of responsive design. However, native applications may offer functionality that cannot be provided in browser environments, including downloading videos for offline viewing. The authors also reported that a hybrid application, through openSAP, a hybrid application, has the advantage of combining native functionalities and web technologies in order to find a balance between maintainability, costs and user experience.

In [9], the authors argue that to create a mobile experience, there are always good reasons to adopt the two strategies, namely to create a native application and to create an optimized mobile website or a web application. They report that native apps are popular because they provide a native user experience, provide access to the hardware capabilities of mobile devices, and leverage the computing power of mobile devices. But the strategy of the native application requires the development of many applications as mobile platforms, which generates a tedious effort in terms of development times, diversified and specialized skill requirements. Regarding web apps, the authors report that despite the existence of tools that promise to reproduce the effects of native operation in the mobile browser, it is still not easy to obtain it. In addition, some features cannot be obtained through a mobile browser such as:

- Access to the hardware functions of mobile devices like integrated sensors, camera, etc.

- Access to mobile databases as SMS, address book, etc.

- The impossibility of running processes in the background in a mobile browser.

In this paper, we present an analysis of the problem of choosing the mobile solution to be implemented in a MOOC/SPOC as a decision-making problem among multiple solutions that can not be incontestably sorted from best to worst. We believe it is difficult to find an "optimal" solution as the decision involves a compromise between several conflicting objectives / criteria which are not perceived in the same manner by all decision makers. We suggest to consider this situation as a multi-objective optimization problem [10].

\section{B. MOOC Providers and the Mobile Access Dimension}

Early MOOC platforms were designed for exclusive access via desktop computers and the size of the mobile access was not taken into account [11]. However, with the rise of mobile technologies and their ubiquity in society, it has become imperative for MOOC providers to rethink the design of their platforms and have a mobile strategy to meet the needs of different learners, especially those of the mobile generation. The majority of MOOC platform providers such as Edx, Coursera, Moodle, Udacity and Iversity, have started to integrate the mobile dimension by offering either platforms with a responsive design that adapts to different mobile browsers and mobile device features, or dedicated native mobile applications that allow mobile access to their MOOC platforms [12]. According to [12], Coursera is ahead of the game in this area. Already in early 2014, it offered native applications addressing both $\mathrm{iOS}$ and Android platforms. Udacity also had two apps, for iOS and Android, but from 2019, both apps were removed from the App store and Google Play. This MOOC provider reports that it wants to focus more on improving the user experience on the desktop while offering a responsive design platform that can be adapted to all devices of different sizes. EdX has caught up with the mobile strategy and has proposed a mobile application starting in 2016 targeting the iOS platform and later Android.

In Table I, we present the main MOOC providers and their different mobile strategies. 
TABLE. I. CONSIDERATION OF THE MOBILE DIMENSION BY THE MAIN MOOCS PLATFORM PROVIDERS

\begin{tabular}{|c|c|c|c|c|}
\hline \multirow{2}{*}{ MOOC provider } & \multirow{2}{*}{$\begin{array}{l}\text { Mobile } \\
\text { web site }\end{array}$} & \multirow{2}{*}{$\begin{array}{l}\text { Responsive } \\
\text { web design }\end{array}$} & Mobile app & \multirow{2}{*}{ Supported mobile OS } \\
\hline & & & Yes/No & \\
\hline Edx www.edx.org & No & Yes & Yes & iOS/Android \\
\hline Udacity www.udacity.com & No & Yes & No & $\begin{array}{l}\text { Available before } 2019 \text { on iOS and Android but from 2019, the } 2 \\
\text { apps have been removed from App store and Google Play }\end{array}$ \\
\hline Coursera www.coursera.org & No & Yes & Yes & iOS/Android \\
\hline Udemy www.udemy.com & Non & Yes & Yes & iOS/Android \\
\hline FutureLearn www.futurelearn.com & No & Yes & No & \\
\hline Moodle www.moodle.org & No & Yes & Yes & iOS/Android \\
\hline Khan Academy www.khanacademy.org & No & Yes & Yes & iOS/Android \\
\hline Rwaq (Saoudi Arabia) www.rwaq.org & No & Yes & Yes & iOS/Android \\
\hline $\begin{array}{l}\text { uc@MOOC mooc.uca.ma } \\
\text { (Cadi Ayyad University -Marrakech) }\end{array}$ & No & Yes & Yes & Android \\
\hline Edraak JORDAN https://www.edraak.org & No & Yes & Yes & iOS/Android \\
\hline
\end{tabular}

\section{THE DIFFERENT APPROACHES FOR THE MOBILE DEVELOPMENT}

In the mobile world, the most popular approaches to mobile development are:

\section{A. Responsive Web Designing (RWD)}

In 2010, Ethan Marcotte introduced the concept of "Responsive Architecture" which means the ability of a physical space to adapt according to the presence of people passing through that space. This concept was later extended to the field of web design and currently refers to the ability of a website to automatically identify the display width of a page and to make the appropriate settings and adjustments necessary to guarantee a display adapted to each user's navigation device [13]. Thus, thanks to the techniques of the reactive Web, it is possible to create websites which adapt and optimize their content to the different navigation devices used by the user in order to improve their user experience whatever the terminal used. Indeed, these techniques allow:

- Adapting the layout to the different screen sizes for both large screen desktops and small phones;

- Automatically readjusting the content according to the width and / or height of the screen on which it is viewed;

- Resizing or changing the location of the images according to the screen resolution;

- Adjusting the structure and elements of the pages so that they can be viewed properly and without zooming on small screens such as those on smartphones or tablets;

- Hiding the elements which are not essential on screens having a small size;

- Making the links and buttons user-friendly for mobile users;

- And respecting users' preferences and personal parameters such as geolocation, device orientation, etc.
Developing a MOOCs / SPOCs platform with a responsive design has the advantage first of all by reducing the cost of development since it is a question of having a single website and offering the same content. Therefore, whatever the device used to follow the MOOCS/SPOCs, the user will have the same experience and follow the same content. In this case, it is not required to master several learning environments of the same MOOC/SPOC. This option also makes maintenance easier and avoids the risk of duplicate and different content.

On the other hand, the referencing of the platform in the search engine results page (SERP) of search engines is kept since it is the same and unique URL of the platform.

A platform of MOOCs/SPOCs in responsive design nevertheless has some drawbacks. We mainly cite the content download time which is longer on mobile devices. Indeed, with Responsive Design, all the elements making up a page are loaded, which has the consequence of reducing the performance of the MOOC/SPOC site on mobile devices.

Responsive Design is an interesting One Web approach if we want the MOOC/SPOC platform to be present on all terminals: Desktop, tablet, television, Smartphone, etc. and especially allows distributing a single and same content on all of these interfaces by reducing development costs while improving the user experience on mobile devices.

\section{B. The Mobile Website}

Mobile websites are another alternative for offering the content of an ergonomic MOOC/SPOC suitable for mobile devices with small screens. It is an approach which consists in developing a mobile site independent of the classic MOOC/SPOC website and which offers its own content, its own functionalities and its own organization. It has been developed so that its ergonomics are simple and suitable for mobile phones with short and targeted content for users. Touch navigation should be optimized through the use of icons to make it easier to use on mobile.

- A mobile site is accessible from the web browser, by entering the site's URL in the navigation bar, hence the need for an internet connection to be able to access it. 
- There are different approaches for the development of a mobile website which can be considered as the light version of a standard website:

- The "mobile first" approach, which consists of designing the mobile version of a site as a priority and then gradually adapting it to larger screens by adding effects and functionalities.

- The classic approach, which is contrary to the concept of "mobile first" because it consists of first developing a standard website and then gradually adapting it to accommodate display on smaller mobile screens by removing effects and functionality which risks degrading the website.

With the improvement of the user-friendliness of mobile browsers, notably Mobile Safari on the iPhone since its first release in 2007 combined with the evolution of website design and development kits (HTML, JavaScript, CSS), display web pages and the readability of their content on small mobile screens has been remarkably improved, especially with the introduction of particular mobile functions such as touch actions, zoom, icons, etc. [14]. Consequently, "web applications", or "web apps" are sites created exclusively for mobiles and use the mobile browser as a runtime environment, thereby taking advantage of the browser's good support for mobile platforms. The "web apps" provide interactivity and navigation adapted for mobiles and an operation which is intended to be close to native mobile applications. The strategy of developing a mobile site enables the deployment of the content of a MOOC/SPOC on the various mobile platforms equipped with an internet browser. This has the advantage of offering redesigned content in order to adapt it to mobile platforms and more suitable functionalities for mobile interfaces. Web applications do not require installation or subsequent upgrades; updates are transparent to the end user. However, this approach also has drawbacks or constraints, in particular: the need for additional work to rethink the content to be deployed on mobile:

- The need for additional content development time to adapt it to mobile,

- The consequent double maintenance of two different environments of the same MOOC/SPOC,

- The need for the user to master two different learning environments depending on the terminal he uses to access the same MOOC/SPOC.

- Another problem is the additional time required to render web pages on mobile and the additional cost required to download the web page from the Internet.

- The need for a permanent internet connection to allow the user to follow a MOOC/SPOC on mobile [15].

- On the other hand, Web applications do not offer full access to the native functionality of smartphones and allow only limited access to the hardware and data of the mobile device; even if today, many software libraries promise to develop Web applications simulating the functionalities of native applications, such as JQuery Mobile, Sencha Touch, etc. In addition, the HTML5 standard promises access to the hardware and software components of the device through a number of APIs (Application Programming Interface).

\section{Mobile Applications (Mobile App)}

A mobile application or "mobile app" is a full-fledged program that installs and runs through the operating system of a mobile phone. It can be installed using an installation file directly on mobile or downloaded by the user via an online store such as App store, Google Play, Windows Phone Store or BlackBerry App World for free or for a fee. Once installed, a mobile app is permanently present on the mobile device and its execution does not generally require an internet connection, apart from certain mobile applications which are developed in connected mode.

The development of mobile applications has evolved with the development of the mobile device market since 2007, notably Apple's famous smartphones and iPhones, for which the first mobile applications were enormously successful both technically and commercially. But from 2010, mobile applications for the Android system began to experience a strong evolution [16]. For example, at the start of 2017, the App Store had around 2.2 million applications compared to 3.6 million applications listed in Google Play.

A mobile application is adapted to mobile screens and offers functionalities, ergonomic and graphic rendering that can be very advanced. Mobile applications offer simple use and navigation that is very suitable for mobile terminals characterized mainly by their small size.

Mobile apps are also marking another revolution with the mobile Internet. Indeed, the mobile Internet allows a mobile application to be embedded in the mobile, that is to say that the content is stored locally on the mobile. They can thus be instantly accessible or refreshed dynamically via network and server connections if the application is running in "connected" mode.

1) Types of mobile application: Mobile development is still faced with the dilemma of choosing between two alternatives, namely:

a) Native mobile applications that target a specific mobile platform such as IOS, Android, Microsoft, Windows Mobile, Symbian, BlackBerry, etc.

b) And hybrid applications which consist of developing a single code (Once code Principle) in standard web technologies (HTML5, JavaScript and CSS) and exploiting it on various existing mobile platforms.

2) Native development: The development of a native application means a specific development for each mobile OS (iOS, Android, Windows phone, etc.). This approach thus strongly depends on the target platform and imposes particular constraints such as the use of Integrated Development Environments (IDE), API (Application Programming Interface) and specific programming languages for each mobile platform. In Table II, we cite the different skills required in terms of development languages to be used according to the targeted mobile platforms. 
TABLE. II. DEVELOPMENT SKILLS CORRESPONDING TO DIFFERENT MOBILE PLATFORMS

\begin{tabular}{|l|l|}
\hline Mobile operating system & Required languages \\
\hline Apple IOS & Objective C, Swift \\
\hline Google Android & Java, kotlin \\
\hline Symbian & $\mathrm{C}++$, Python, HTML/CSS/JS \\
\hline Windows mobile, Windows 7 Phone & $\mathrm{C}++$, C\# \\
\hline Blackberry (ex RIM) & Java \\
\hline Samsung bada & $\mathrm{C}++$ \\
\hline
\end{tabular}

3) Hybrid development: A hybrid application is a mobile application that operates halfway between a native application and a web app. Like native applications, it downloads from a store, launches like native applications and does not require an internet connection. It runs like a web application in a browser with the difference that the browser is part of the hybrid application, unlike a web app whose source code is always downloaded from the web.

4) Advantages and limitations: Hybrid applications are primarily designed using web technologies such as HTML5, JavaScript and CSS. Since 2015, the market for hybrid applications has grown considerably, especially with the evolution of mobile development frameworks that provide impressive results in terms of design, functionality and user experience very close to that obtained with native development.

The native application development process is the right way to deploy mobile-ready applications that deliver a rich user experience, fast performance, consistent look and full access to mobile platform hardware and data. Indeed, the unique technology environment of native applications enables the exploitation of native mobile functionality and takes full advantage of the specificity of mobile devices. For example, a native:

- Allows a better integration with the mobile system interface (pixel-accurate positions of the action buttons at the bottom of the screen, virtual keyboard, etc.);

- Offers the ability to use the mobile's hardware devices (GPS, camera, etc.);

- Provides interaction with "contacts, calendar, SMS/MMS functions" etc.

- Makes it possible to exploit alert and notification functions that are particularly symbolic of the advanced capabilities of mobile applications,

- And permits to take into account local user settings such as default language, portrait or landscape display mode offering the most personal user experience on the mobile.

However, there are disadvantages to this approach. First of all, it requires advanced technical skills and inevitably leads to an increase in development time because it is impossible to reuse the source code produced for one platform on another, since each system requires its own code. This leads to an increase in maintenance costs, which represents a real challenge for the native development approach.

As far as hybrid development is concerned, this approach allows single code writing and does not require any detailed knowledge of the target platform so that allows a multiplatform deployment. This makes it a very attractive model for several reasons, including the advantage of optimizing development time and effort, simplifying maintenance and deployment processes [17].

Despite the obvious advantages of hybrid mobile applications, there are also some disadvantages, such as limited access to the hardware features of mobile devices and reduced performance, especially in terms of speed and user experience as with native applications [17].

\section{COMPARATIVE ANALYSIS BETWEEN THE DIFFERENT APPROACHES TO MOBILE INTEGRATION IN A MOOC/SPOC}

In Table III, we present a summary of the different strategies for integrating the mobile dimension in a MOOC/SPOC and some elements of comparison and decisionmaking criteria.

\section{A Multi-Objective Optimization Analysis to Choose THE MobILE SOLUTION}

To set up a mobile strategy in a MOOC/SPOC, it appears that wanting to increase the performance of the mobile solution increases development time (Fig. 1). We are therefore faced with a dilemma where we are unable to improve the Performance criterion without deteriorating the development time criterion. This is typical of multi-objective optimization problems that consist in looking for the best compromise between the different objectives to be optimized [10]. For our analysis, we have chosen a rating scale from 1 to 5 to indicate the smallest to the greatest degree (Table IV).

Formally, a multi-objective optimization problem is a problem described as follows:

Minimize $F(x)=\left(f_{1}(x), f_{2}(x), \ldots \ldots, f_{m}(x)\right)$ for $x \in X$ and knowing that $\mathrm{g}_{\mathrm{k}}(\mathrm{x}) \leq 0$. Where:

- $\mathrm{x}=\left(\mathrm{x}_{1}, \mathrm{x}_{2}, \ldots, \mathrm{x}_{\mathrm{n}}\right)$ is the vector of $\mathrm{n}$ feasible solutions $\mathrm{x}_{\mathrm{i}}$ with $1 \leq \mathrm{i} \leq \mathrm{n}$

- $\mathrm{x} \in \mathrm{X}$ the set of solutions

- $\mathrm{F}(\mathrm{x})$ is the objective vector, where $m$ is the number of objective functions with $m \geq 2$

- $\mathrm{f}_{\mathrm{j}}$ is an objective to be optimized (minimized or maximized) where $1 \leq \mathrm{j} \leq \mathrm{m}$ knowing that maximizing $f_{j}$ means minimizing $-f_{j}$

- $g_{k}$ represents a constraint that limits the values that solutions can take, $\mathrm{g}_{\mathrm{k}}(\mathrm{x}) \leq 0$ where: $1 \leq \mathrm{k} \leq \mathrm{p}$.

The solution of a multi-objective problem is not unique, but there is a set of solutions called "non-dominated set". These solutions are those where improvement in one objective systematically leads to deterioration in the performance of at least one other objective. 
TABLE. III. COMPARISON OF THE DIFFERENT APPROACHES FOR THE DEVELOPMENT OF THE MOBILE DIMENSION IN A MOOC/SPOC

\begin{tabular}{|c|c|c|c|c|}
\hline Criterion & Native mobile app & Hybrid mobile app & Web app & Responsive design website \\
\hline Development & $\begin{array}{l}\text { Code specific to each } \\
\text { target platform }\end{array}$ & Unique code & $\begin{array}{l}\text {-Technologies of the Web (HTML / } \\
\text { Javascript / CSS) }\end{array}$ & LMS + responsive design theme \\
\hline $\begin{array}{l}\text { Skills required for } \\
\text { mobile development }\end{array}$ & Highly advanced & advanced & Moderately advanced & $\begin{array}{l}\text { Some settings at the LMS level } \\
\text { - }\end{array}$ \\
\hline Development time & $\begin{array}{l}\text { Very long } \\
\text { (Double) }\end{array}$ & $\begin{array}{l}\text { Long } \\
\text { (double) }\end{array}$ & $\begin{array}{l}\text { Medium to long } \\
\text { (Double) }\end{array}$ & Medium \\
\hline $\begin{array}{l}\text { Compatibility on } \\
\text { platforms }\end{array}$ & $\begin{array}{l}\text { Compatible on a single } \\
\text { platform }\end{array}$ & $\begin{array}{l}\text { Compatible on several } \\
\text { platforms }\end{array}$ & online & online \\
\hline Deployment & On a store & On a store & On Internet & On Internet \\
\hline Need for Installation & Yes & Yes & No & No \\
\hline Offline availability & Yes & Yes & No & No \\
\hline $\begin{array}{l}\text { Access to mobile } \\
\text { equipment and data }\end{array}$ & Yes & Yes & Yes but limited & No \\
\hline UX user experience & Yes complete & Yes but limited & $\begin{array}{l}\text { Simulated Can be Enhanced by APIs } \\
\text { /libraries Same ergonomics for all } \\
\text { platforms }\end{array}$ & Web responsive \\
\hline Performance & Native / custom & Simulated / Limited & $\begin{array}{l}\text { Reduced Depends on the quality of the } \\
\text { network, Depends on the browser } \\
\text { Requires more Code and Resource } \\
\text { Download Time }\end{array}$ & $\begin{array}{l}\text { Low (time to download } \\
\text { resources (videos, pictures, } \\
\text { etc.)). }\end{array}$ \\
\hline Distribution & $\begin{array}{l}\text { On a store. Requires a } \\
\text { validation at each update }\end{array}$ & $\begin{array}{l}\text { On a store. Requires a } \\
\text { validation at each update }\end{array}$ & On the web & On the web \\
\hline Updates & requires reinstallation & requires reinstallation & Transparent & Transparent \\
\hline Maintenance & Double & Double & Double & One site to maintain \\
\hline $\begin{array}{l}\text { MOOC/SPOC } \\
\text { referencing }\end{array}$ & $\begin{array}{l}\text { A mobile application + } \\
\text { A site for the } \\
\text { MOOC/SPOC }\end{array}$ & $\begin{array}{l}\text { A mobile application }+ \\
\text { A site for the MOOC/SPOC }\end{array}$ & $\begin{array}{l}\text { Two URLs: one for the mobile site and } \\
\text { one for the MOOC/SPOC site. }\end{array}$ & $\begin{array}{l}\text { A unique URL for the } \\
\text { MOOC/SPOC site. }\end{array}$ \\
\hline Price to market & Hight+ & Hight+ & Hight & Medium \\
\hline
\end{tabular}

TABLE. IV. RATING SCALE

\begin{tabular}{|l|l|}
\hline \multicolumn{2}{|l|}{ Rating scale } \\
\hline 5 & Very good \\
\hline 4 & Well \\
\hline 3 & Medium \\
\hline 2 & Bad \\
\hline 1 & Very bad \\
\hline
\end{tabular}

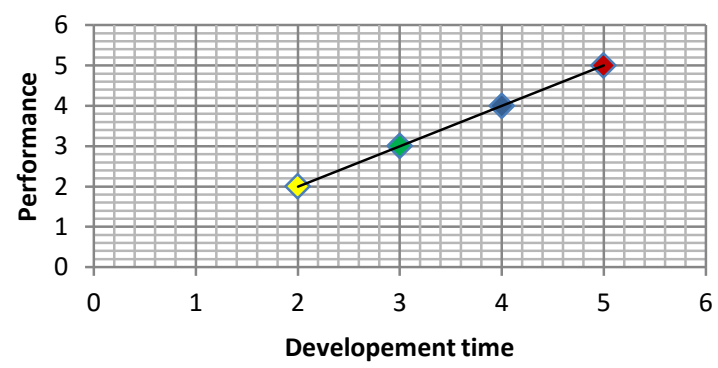

Fig. 1. Mobile Solutions: Performance vs. Development Time.
In the next section, we formulate the problem of choosing the mobile solution to be implemented in a MOOC/SPOC as an optimization problem with several objectives.

\section{Description OF THE PROBLEM OF CHOOSING THE MOBILE STRATEGY IN A SPOC}

The problem of choosing the mobile strategy in a MOOC/SPOC can be described as follows:

- $\mathrm{X}$ A set of the mobile solutions $\mathrm{X}=\left(\mathrm{x}_{1}, \mathrm{x}_{2}, \mathrm{x}_{3}, \mathrm{x}_{4}\right)$

- $\mathrm{x}_{1}$ : Native Mobile app (NM);

- $\mathrm{x}_{2}$ : Hybrid Mobile app (HM);

- $\mathrm{x}_{3}$ : Mobile Website (MW)

- $\mathrm{x}_{4}$ : Responsive Website (RW).

- The Several objectives to minimize or maximize: $\mathrm{f}_{\mathrm{j}}(\mathrm{x})$ where: $1 \leq \mathrm{j} \leq 6$

- $\mathrm{f}_{1}(\mathrm{x})$ : Development time to minimize (DT)

- $\mathrm{f}_{2}(\mathrm{x})$ : Performances to maximize (PR) (which is equivalent to minimize $-f_{2}(x)$ 
- $\mathrm{f}_{3}(\mathrm{x})$ : User experience to maximize (UX)

- $\mathrm{f}_{4}(\mathrm{x})$ : Maintenance to be minimized (MN)

- $\mathrm{f}_{5}(\mathrm{x})$ : Update Transparent to the user to maximize (or minimize according to the designer's vision) (UT)

- $\mathrm{f}_{6}(\mathrm{x})$ : Offline access to maximize or minimize depending on the designer's vision $(\mathrm{OF})$

For illustrative purposes, Table $\mathrm{V}$ provides an example of the costs of the different criteria selected for each mobile solution on a scale of 1 to 5 .

\section{A. Illustration for Two Objectives}

We will find the Pareto dominance relations [18] of the solutions $\mathrm{x}_{\mathrm{i}}$ for the case of the following two objectives:

- The Development Time $f_{1}$ (to be minimized)

- The performance $f_{2}$ (to be maximized) which is equivalent to minimizing $-f_{2}$. (Inverse notation from 5 to 1 : from the weakest to the strongest)

Let's call that a feasible solution $\mathrm{x} \in \mathrm{X}$ is "optimal Pareto" [19] or "not dominated" if and only if there is no solution $y \in X$ such that $\mathrm{y}$ dominates $\mathrm{x}$.

It is said that a solution $\mathrm{y}$ "dominates" a solution $\mathrm{z}$ in the case of goal minimization, if and only if:

$\forall \mathrm{i} \in[1 . . \mathrm{m}], \mathrm{f}_{\mathrm{i}}(\mathrm{y}) \leq \mathrm{f}_{\mathrm{i}}(\mathrm{z})$ and $\exists \mathrm{j} \in[1 . . \mathrm{m}]$ such as

$\mathrm{f}_{\mathrm{j}}(\mathrm{y})<\mathrm{f}_{\mathrm{j}}(\mathrm{z})$.

Thus, any solution in the Pareto set can be considered optimal since no improvement can be made on one objective without degrading the relative value of another objective. These solutions form what is called "the Pareto front".

In Fig. 2, we represent the case of the two-objective problem taken for illustration. We can notice that the four moving alternatives form the "Pareto front" since they are all non-dominated solutions.

TABLE. V. OBJECTIVES TO Minimize

\begin{tabular}{|l|l|l|l|l|l|l|}
\hline Mobile Solution/Objective & $\mathbf{f}_{\mathbf{1}}$ & $-\mathbf{- f}_{\mathbf{2}}$ & $-\mathbf{f}_{\mathbf{3}}$ & $\mathbf{f}_{\mathbf{4}}$ & $-\mathbf{f}_{\mathbf{5}}$ & $-\mathbf{- f}_{\mathbf{6}}$ \\
\hline NM & 5 & 1 & 1 & 5 & 5 & 1 \\
\hline HM & 4 & 2 & 2 & 3 & 5 & 1 \\
\hline MW & 3 & 3 & 3 & 3 & 1 & 5 \\
\hline RW & 2 & 4 & 4 & 1 & 1 & 5 \\
\hline
\end{tabular}

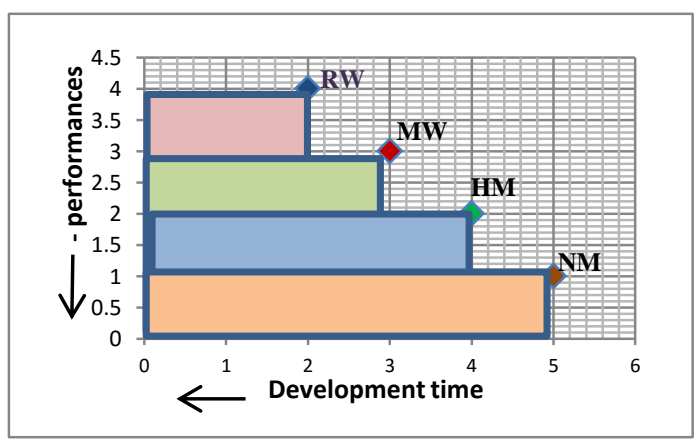

Fig. 2. Non Dominated Mobile Solutions.

\section{B. Multi-Objective Case}

In Fig. 3, we have represented the mobile solutions according to their ratings in relation to the different objectives [1] we have considered.

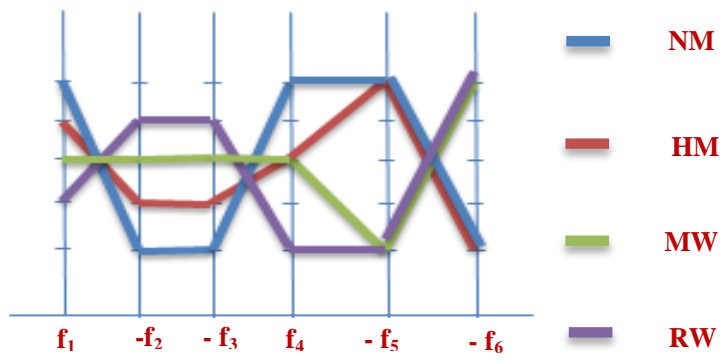

Fig. 3. Case of Several Objectives to be Minimized.

By comparing the solutions two by two, we can note that no one solution dominates the other:

- NM, HM: The native mobile solution minimizes $-\mathrm{f}_{2}$ but maximizes $\mathrm{f}_{1}$

- HM, NM: Mobile hybrid solution minimizes $\mathrm{f}_{4}$ but maximizes $-\mathrm{f}_{3}$

- NM, MW: Native mobile solution minimizes $-\mathrm{f}_{2}$ but maximizes $\mathrm{f}_{1}$

- MW, NM: Mobile web solution minimizes $f_{1}$ but maximizes $-\mathrm{f}_{2}$

- NM, RW: Native mobile solution minimizes $-\mathrm{f}_{2}$ but maximizes $\mathrm{f}_{1}$.

- RW, NM: Responsive web solution minimizes $\mathrm{f}_{4}$ but maximizes $-\mathrm{f}_{3}$.

- HM, MW: Mobile hybrid solution minimizes $-\mathrm{f}_{2}$ but maximizes $-\mathrm{f}_{5}$.

- MW, HM: Mobile web solution minimizes $\mathrm{f}_{1}$ but maximizes $-\mathrm{f}_{3}$.

- HM, RW: Mobile hybrid solution minimizes $-\mathrm{f}_{2}$ but maximizes $\mathrm{f}_{4}$.

- RW, HM: Responsive site solution minimizes $f_{1}$ but maximizes $-\mathrm{f}_{3}$.

- MW, RW: Mobile site solution minimizes $-\mathrm{f}_{2}$ but maximizes $\mathrm{f}_{4}$.

- RW, MW: Responsive site solution minimizes $\mathrm{f}_{4}$ but maximizes $-f_{3}$.

Therefore, the four mobile solutions form an optimal set of solutions since no improvement can be made on one objective without degrading the relative value of another objective.

\section{VII.CONCLUSION}

Today, strengthening its presence on mobile is no longer an option but rather a necessity because the mobile has become an essential tool to access many services. MOOCs/SPOCs designers are also concerned with this challenge and must consider a solution to target all users, whatever the device used for access: desktop, laptop, smartphone, tablet. 
The various approaches to integrate the mobile dimension in a MOOC/SPOC are different and each solution has advantages and disadvantages from a technical point of view. The choice must be made according to the needs and uses of the end users.

Based on the above analysis, we can admit with certainty, that the problem of choosing the mobile solution to be implemented in a SPOC is a problem of decision between several solutions that cannot be unquestionably ordered from the best to the worst. Moreover, it is difficult to find an "optimal" solution since the decision is a compromise between several conflicting objectives/criteria and this compromise is not seen in the same way by all decision-makers. We can thus consider this situation as a multi-objective optimization problem and in the present work. We found that the four mobile solutions proposed constitute an optimal set of non dominated solutions since no improvement can be made on one objective without degrading the relative value of another objective.

\section{REFERENCES}

[1] Sawaragi, Y., Nakayama, H., \& Tanino, T. (Eds.). (1985). Theory of multiobjective optimization. Elsevier.

[2] Sener, O., \& Koltun, V. (2018). Multi-task learning as multi-objective optimization. Advances in Neural Information Processing Systems (pp. 527-538).

[3] Mirjalili, S., Jangir, P., \& Saremi, S. (2017). Multi-objective ant lion optimizer: a multi-objective optimization algorithm for solving engineering problems. Applied Intelligence, 46(1), 79-95.

[4] Ejday, M. (2011). Optimisation Multi-Objectifs à base de Métamodèle pour les Procédés de Mise en Forme (Doctoral dissertation).

[5] Irisarri, F. X., Bassir, D. H., Carrere, N., \& Maire, J. F. (2009). Multiobjective stacking sequence optimization for laminated composite structures. Composites Science and Technology, 69(7-8), 983-990.

[6] De Waard, I., Koutropoulos, A., Hogue, R. J., Abajian, S. C., Keskin, N. Ö., Rodriguez, C. O., \& Gallagher, M. S. (2012). Merging MOOC and mLearning for Increased Learner Interactions. International Journal of Mobile and Blended Learning, 4(4), 34-46. doi:10.4018/ jmbl.2012100103.
[7] Sharples, M., Delgado Kloos, C., Dimitriadis, Y., Garlatti, S., \& Specht, M. (2015). Mobile and accessible learning for MOOCs. Journal of interactive media in education, 1(4), 1-8.

[8] Renz, J., Staubitz, T., \& Meinel, C. (2014). MOOC to Go. International Association for the Development of the Information Society.

[9] Harb, E., Kapellari, P., Luong, S., \& Spot, N. (2011). Responsive web design. Version 6.

[10] Gagné, C., Gravel, M., \& Price, W. L. (2004). Optimisation multiobjectifs a l'aide d'un algorithme de colonie de fourmis. INFOR: Information Systems and Operational Research, 42(1), 23-42.

[11] Dalipi, F., Imran, A. S., Idrizi, F., \& Aliu, H. (2016). An Analysis of Learner Experience with MOOCs in Mobile and Desktop Learning Environment. Advances in Intelligent Systems and Computing, 393402. doi:10.1007/978-3-319-42070-7_36.

[12] Taneja, S., \& Goel, A. (2014). MOOC providers and their strategies. International Journal of Computer Science and Mobile Computing, 3(5), 222-228.

[13] Peng, W., \& Zhou, Y. (2015, July). The Design and Research of Responsive Web Supporting Mobile Learning Devices. In 2015 International Symposium on Educational Technology (ISET) (pp. 163167). IEEE.

[14] Godwin-Jones, R. (2011). Mobile apps for language learning. Language Learning \& Technology, 15(2), 2-11.

[15] Xanthopoulos, S., \& Xinogalos, S. (2013, September). A comparative analysis of cross-platform development approaches for mobile applications. In Proceedings of the 6th Balkan Conference in Informatics (pp. 213-220). ACM.

[16] Dekhane, S., \& Tsoi, M. (2012). Designing a Mobile Application for Conceptual Understanding: Integrating Learning Theory with Organic Chemistry Learning Needs. International Journal of Mobile and Blended Learning, 4(3), 34-52. doi:10.4018/ jmbl.2012070103.

[17] Malavolta, I., Ruberto, S., Soru, T., \& Terragni, V. (2015, May). Hybrid mobile apps in the google play store: An exploratory investigation. In Proceedings of the second acm international conference on mobile software engineering and systems (pp. 56-59). IEEE Press.

[18] Marler, R. T., \& Arora, J. S. (2004). Survey of multi-objective optimization methods for engineering. Structural and multidisciplinary optimization, 26(6), 369-395.

[19] Pareto, V. (1964). Cours d'économie politique (Vol. 1). Librairie Droz. 